OPEN ACCESS

Edited by:

Ying $X u$,

University at Buffalo, United States

Reviewed by:

Shanbao Tong,

Shanghai Jiao Tong University, China Long Meng,

Shenzhen Institutes of Advanced

Technology, Chinese Academy

of Sciences (CAS), China

*Correspondence:

Yang Zhang

drzhy001@163.com

Weidong Zhou

wdzhou@sdu.edu.cn

Received: 20 January 2021 Accepted: 02 March 2021 Published: 15 April 2021

Citation:

Liu Y, Wang G, Cao C, Zhang G, Tanzi EB, Zhang Y, Zhou W and Li Y (2021) Neuromodulation Effect of Very Low Intensity Transcranial Ultrasound

Stimulation on Multiple Nuclei in Rat

\section{Neuromodulation Effect of Very Low Intensity Transcranial Ultrasound Stimulation on Multiple Nuclei in Rat Brain}

\author{
Yingjian Liu', Gang Wang ${ }^{1}$, Chao Cao ${ }^{1}$, Gaorui Zhang ${ }^{2,3}$, Emily B. Tanzi ${ }^{4}$, Yang Zhang ${ }^{2 *}$, \\ Weidong $\mathrm{Zhou}^{1 *}$ and $\mathrm{Yi} \mathrm{Li}^{4}$ \\ ${ }^{1}$ School of Microelectronics, Shandong University, Jinan, China, ${ }^{2}$ Department of Radiology, Qilu Hospital of Shandong \\ University, Jinan, China, ${ }^{3}$ School of Medical Imaging, Weifang Medical University, Weifang, China, ${ }^{4}$ Weill Cornell Medicine, \\ New York, NY, United States
}

Objective: Low-intensity transcranial ultrasound stimulation (TUS) is a non-invasive neuromodulation technique with high spatial resolution and feasible penetration depth. To date, the mechanisms of TUS modulated neural oscillations are not fully understood. This study designed a very low acoustic intensity (AI) TUS system that produces considerably reduced Al Ultrasound pulses (ISPTA $<0.5 \mathrm{~W} / \mathrm{cm} 2)$ when compared to previous methods used to measure regional neural oscillation patterns under different TUS parameters.

Methods: We recorded the local field potential (LFP) of five brain nuclei under TUS with three groups of simulating parameters. Spectrum estimation, time-frequency analysis (TFA), and relative power analysis methods have been applied to investigate neural oscillation patterns under different stimulation parameters.

Results: Under PRF, $500 \mathrm{~Hz}$ and $1 \mathrm{kHz}$ TUS, high-amplitude LFP activity with the autorhythmic pattern appeared in selected nuclei when ISPTA exceeded $12 \mathrm{~mW} / \mathrm{cm}^{2}$. With TFA, high-frequency energy (slow gamma and high gamma) was significantly increased during the auto-rhythmic patterns. We observed an initial plateau in nuclei response when ISPTA reached $16.4 \mathrm{~mW} / \mathrm{cm}^{2}$ for RPF $500 \mathrm{~Hz}$ and $20.8 \mathrm{~mW} / \mathrm{cm}^{2}$ for RPF $1 \mathrm{kHz}$. The number of responding nuclei started decreasing while ISPTA continued increasing. Under $1.5 \mathrm{kHz}$ TUS, no auto-rhythmic patterns have been observed, but slow frequency power was increased during TUS. TUS inhibited most of the frequency band and generated obvious slow waves (theta and delta band) when stimulated at RPF $=1.5 \mathrm{kHz}$, $I_{S P T A}=8.8 \mathrm{~mW} / \mathrm{cm}^{2}$.

Conclusion: These results demonstrate that very low intensity Transcranial Ultrasound Stimulation (VLTUS) exerts significant neuromodulator effects under specific parameters in rat models and may be a valid tool to study neuronal physiology.

Keywords: transcranial ultrasound stimulation, local field potentials, neuromodulation, hippocampus, ephaptic coupling, neuronal resonance, synchronization 


\section{INTRODUCTION}

Low-intensity transcranial ultrasound stimulation (TUS) is a non-invasive neuromodulation technique that can be reliably transmitted through the skull to stimulate neurons with high spatial resolution and deep penetration depth (Hayner and Hynynen, 2001). TUS could change behavioral and electrophysiological processes by stimulating particular regions of the brain, (Yoo et al., 2011) and it has been proved an effective tool in clinical neurosciences (Tufail et al., 2010). Recent studies show that TUS has much neurotherapeutic potential in ischemic brain injury, (Guo et al., 2015) epilepsy, (Hakimova et al., 2015) depression, (Zhang et al., 2018), and Alzheimer disease (Lin et al., 2015).

Neuromodulation has been implemented broadly in neuroscience research, with several methods developed in recent years. Electrical methods such as deep-brain stimulation (DBS) have a high targeting spatial resolution and are considered highly effective therapies for disease intervention. But DBS suffers limitations such as invasiveness, and brain impalement with electrodes. Transcranial magnetic stimulation (Barker, 1999; Perera et al., 2016) does not require surgery but suffers from low spatial resolution. Optogenetic-based approaches have extraordinary spatial precision but require genetic manipulation. These kinds of methods have inevitably limited applications. In addition to the aforementioned methods, TUS is a promising non-invasive neuromodulation technology with better spatial resolution and higher penetration depth (Hayner and Hynynen, 2001). Many studies were conducted to measure the effects of ultrasound stimulation on neural oscillations ex vivo or in vivo (Bystritsky et al., 2011). In contrast to high intensity US, low-intensity US ( $I_{S P P A}: 0.5-100 \mathrm{~W} / \mathrm{cm}^{2}$ ), is delivered in a pulsed mode for brief periods of time, has been widely used in animal neuromodulation studies and can directly stimulate action potentials and synaptic transmission through mechanisms involving the non-thermal activation of ion channels (Tyler et al., 2008).

Local field potential (LFP) recorded extremely local phenomena within the nuclei, so it can accurately measure the brain function changes after ultrasonic stimulation. Previous studies have recorded LFPs in the motor cortex before and after ultrasound stimulation with different stimulation parameters (Wang et al., 2019), and demonstrates that TUS can significantly decrease parkinsonian-related activity in mice administered MPTP (Wang et al., 2020). By using LFPs to analyze the changes quantitatively, Yuan pointed out that neural activities and hemodynamic response of the mouse visual cortex induced by TUS are related to the ultrasound intensity (Yuan et al., 2021). LPF can also provide electrophysiological recording, which proves that cortical hemodynamics changes induced by TUS are related to stimulation intensity and duration (Yuan et al., 2020). Therefore, we investigate the change of electrophysiological responses across different brain regions under different TUS parameters by using LPFs.

Ultrasound directly activates a localized area, (Tufail et al., 2010) or indirectly activates auditory pathways that, in turn activate other cortical networks (Guo et al., 2018;
Sato et al., 2018). Another study also showed TUS induced hemodynamic responses; however, the acoustic intensity (AI) was much higher in that study than the Food and Drug Administration (FDA) guidelines defined $\left(I_{S P T A} \leq 94 \mathrm{~mW} / \mathrm{cm}^{2}\right)$ (Center for Devices and Radiological Health, 2019). The neuronal membrane is a dedicated structure, different neuronal oscillation pattern may occur under the very low intensity US simulation. Here we designed a very low AI TUS system that can produce much lower AI pulse $\left(I_{S P P A}<0.5 \mathrm{~W} / \mathrm{cm}^{2}\right.$, $I_{S P T A}<100 \mathrm{~mW} / \mathrm{cm}^{2}$ ) to investigate the minimum stimulus intensity of nerve oscillation in different brain regions. What's more, we hope to reduce power consumption of ultrasonic neuromodulation and to reduce retroinhibition by high AI TUS.

Despite the broad application potential of ultrasound, the multi-nucleus mechanisms underlying TUS-modulated neural oscillations remain poorly understood. Most ultrasonic neural regulation is limited to the study of LFP in a single nucleus. In this study, we hope to explore the differences of nuclei under ultrasound stimulation from the perspective of multiple nuclei. Five nuclei, SI, CA1, CA2, LHb, and MI, have been selected in this study to examine different brain networks crossing motor, sensory, perception, and cognition (Rinaldi et al., 1991; Bachtold et al., 1998; Matsumoto and Hikosaka, 2009). These five nuclei are also associated with memory and feeling. Therefore, understanding the response of these nuclei after ultrasonic stimulation can provide a significant value for the ultrasound treatment of many neurological diseases.

In this study, we designed a very low-intensity TUS system $\left(I_{S P T A}<30 \mathrm{~mW}^{2}\right)$ to stimulate the rat brain under different frequencies and AIs. LFP signals of five nuclei were recorded simultaneously and processed by spectrum estimation, timefrequency analysis (TFA), and relative power analysis methods with three sets of parameters at different ultrasonic intensities to investigate the TUS induced neuromodulation. To the best of our knowledge, this study is the first to analyze neuromodulation effects of deep brain nuclei under very low intensity transcranial ultrasound stimulation (VLTUS).

\section{MATERIALS AND METHODS}

\section{Animal Anesthesia and Surgery}

Two adult Sprague-Dawley rats (male: 270-310 g) were purchased from the Laboratory of Animal Center of Zhejiang University. Both rats were anesthetized with chloral hydrate solution $(10 \%, 0.35 \mathrm{ml} / 100 \mathrm{~g})$. The anesthetized rats were fixed on a stereotaxic apparatus (51603U, RWD, China) with ear bars and a clamping device to keep their heads horizontal. Specifically, the fur covering each rat's skull was trimmed to expose the cleaner scalp, and the scalp was sterilized with iodine. The bregma and lambda were explored after cutting the scalp and removing the subcutaneous tissue. One hole was drilled into the rats' meninges to fix reference electrodes. Another hole was drilled at the intersection of the sagittal suture and the interaural line as the ground. Five recorded sites were determined according to the reference (Paxinos and Watson, 2006) (primary sensory cortex, SI: $\mathrm{AP}=-4.44 \mathrm{~mm}, \mathrm{ML}=6 \mathrm{~mm}, \mathrm{DV}=3 \mathrm{~mm}$; primary 
motor cortex, $\mathrm{MI}: \mathrm{AP}=0.48, \mathrm{ML}=2.2, \mathrm{DV}=2$; lateral habenular nu, LHb: $\mathrm{AP}=-2.52, \mathrm{ML}=0.6, \mathrm{DV}=4.8$; field CA1 of the hippocampus, $\mathrm{CA1}$ : $\mathrm{AP}=-3.96, \mathrm{ML}=1.2, \mathrm{DV}=3.4$; field $\mathrm{CA} 2$ of the hippocampus, CA2: $\mathrm{AP}=-5.20, \mathrm{ML}=5.6, \mathrm{DV}=6$ ). Figure 1A shows the anatomy map of the rat. It visualizes the sites of LFP recorded under different ultrasound stimulation parameters. The electrode and the head wound of the rat were sealed with dental cement, and only the interface of the recording electrodes was exposed finally. After the animal surgery, the two rats were housed in cages for a week to recover with free access to food and water. This study's protocols were approved by the Laboratory Animal Ethical and Welfare Committee of Shandong University Cheeloo College of Medicine.

In order to rule out accidental circumstances, we repeated the experiment three times for each stimulus parameter on two adult Sprague-Dawley rats.

\section{TUS Experimental Setup}

In the TUS system, an arbitrary function generator (YB16051A, Lv yang, China) was used to control the pulsed repetition frequency (PRF), fundamental frequency (FF), number of cycles per pulse $(\mathrm{NC} / \mathrm{p})$, and duty cycle (DC) of ultrasound. The pulsed sequence from the generator was amplified by a high voltage amplifier (ATA-2021H, Aigtek, China) and transmitted to an ultrasound transducer (focal length of $45 \mathrm{~mm}$ and focus diameter of $20 \mathrm{~mm}$ ). The ultrasound transducer was connected to the rat skull by a conical acoustic collimator filled with ultrasound coupling gel (Figure 2A). The collimator is a circular area with a radius of $0.4 \mathrm{~cm}$, so the focus area does not exceed $0.51 \mathrm{~cm}^{2}$. The transducer connected to the collimator is $8 \mathrm{~mm}$ higher than the skull, so the focal length into the brain is less than $37 \mathrm{~mm}$. As illustrated in Figure 1A, in order to reduce ultrasonic attenuation, electrodes were implanted into the nucleus vertically, and the collimator was aimed horizontally from the occipital side. The angle between them was $90^{\circ}$. The electrode and the head skull of the rat were all sealed with dental cement. In order to avoid the obstruction and attenuation of ultrasound by dental cement, we applied ultrasound to the occipital lobe. The stimulation site was chosen as shown in Figure 1A, $6.5 \mathrm{~mm}$ lateral of midline and $4 \mathrm{~mm}$ posterior of bregma.

Pulsed ultrasound sequences were constructed as illustrated in Figure 2B. The FF and DC of the ultrasound were $500 \mathrm{kHz}$ and $20 \%$ respectively. By using the collimator, the ultrasonic focal spot radius was fixed at $0.4 \mathrm{~cm}$. Under the condition of fixed FF and DC, we designed three groups of trials by changing PRF and NC/p. For each trial, we changed the magnification of the amplifier to get the increasing AI. Three PRFs $(500 \mathrm{~Hz}, 1,000 \mathrm{~Hz}$, and 1,500$)$ were used in this study. The range of $\mathrm{I}_{S P T A}$ was from 7.2 to $25.6 \mathrm{~mW} / \mathrm{cm}^{2}$. The ultrasound parameters, spatial peak temporal average intensity $\left(\mathrm{I}_{S P P A}\right)$, spatial peak pulse average ( $\left.\mathrm{I}_{S P T A}\right), \mathrm{PRF}$, and NC/p we used are listed in Table $\mathbf{1 .}$

The ultrasonic power generated by the plane transducer (installed on the collimator) was measured by the radiation force method using the ultrasound power meters model (UPM-DT1AV, Ohmic, United States). During this process, the transducer was held above the de-gassed water by a positioning clamp. The ultrasonic energy passed through the water to reflect off the conical target and was then absorbed by the rubber lining. The radiant power was proportional to the total downward force in the conical target. This weight was then transferred to the electro-mechanical load cell, which could produce a digital readout in watts of power. In this experiment we assumed that the brain and tissues had the sound, speed, and density of water. The final acoustic attenuation transmitted into the brain was $83.9 \%$ because of the absorption and refraction properties of the ultrasound in the rat skull. Since the five nuclei are not far apart, the ultrasound intensity at different nuclei is the same. Thus, we only consider the attenuation of a skull. Details of the AI calculation are described in the Appendix.

\section{Data Acquisition}

Five electrodes (nickel-chromium alloy, $35 \mu \mathrm{m}$, A-M SYSTEM, United States) were used to record the LFP signals in the designated brain area of rats. Each electrode was made of two nickel-chrome wires that were intertwined with each other. The reference electrode implanted into the rats' meninges was made in the same way as the recording electrodes. The ground electrode was made of exposed silver wire. Finally, all electrodes were connected to a 16-channel $(2 \times 8$ array $)$ interface.

The raw LFP signals produced in response to TUS were collected by a 32-channel neural signal processor (RHD2132, Intan, United States), which can perform pre-amplification and $\mathrm{AD}$ conversion at the same time. These signals were acquired at the sampling frequency of $1 \mathrm{kHz}$ and stored on field programmable gate array (FPGA). Finally, all data were transmitted to the computer and processed using MATLAB 2018b (MathWorks, Inc., United States).

After the final recording session, the animal was perfused with paraformaldehyde (PFA) 4\%, and histological analysis was performed to confirm electrode locations. Correct electrode position was confirmed for all animals in this study.

\section{Spectral Estimation and Time-Frequency Analysis}

Spectral estimation can transform a signal from the time domain to the frequency domain and provide a description of the signal's power distribution along with frequency (Hu and Zhang, 2019). The foundation of spectral estimation is the discrete Fourier transform (DFT), which is calculated using the Fast Fourier transform (FFT) algorithm for computational efficiency. Taking discrete-time signal $x_{n}, n=1,2 \ldots \mathrm{N}$ as an example, the DFT of $x_{n}$ can be written as

$$
\mathrm{F}(\mathrm{k})=\sum_{n=0}^{N-1} x_{n} e^{-j 2 \pi k n / N}
$$

Based on DFT, the power spectral density (PSD) can be calculated to describe how the power of signal is distributed over different frequencies. In our study, LFP signals were acquired at the sampling frequency of $1 \mathrm{kHz}$ with software designed Bandpass (1-140 Hz, 1-45 Hz) and notch (50, $100 \mathrm{~Hz}$ ) filter. And LFP powers were computed for individual channels using the multitaper spectral estimation technique 

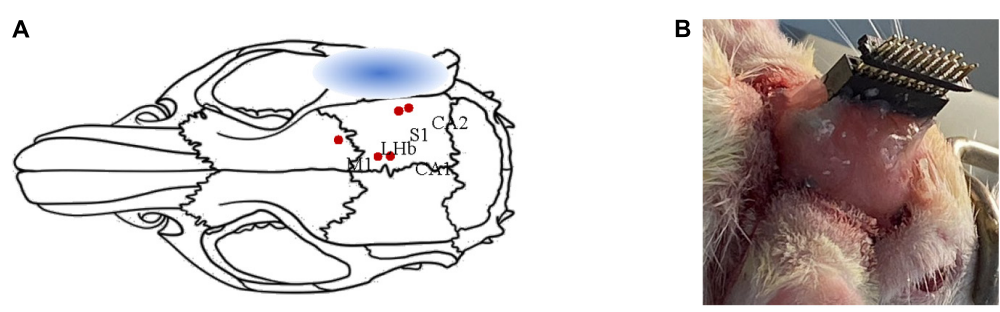

Simulation Site

FIGURE 1 | Schematic diagram of animal surgery. (A) Anatomy map of rat. The relative positions of the five nuclei are marked with red dots. (B) The final form of surgery. The electrodes are sealed only the interface of electrodes were exposed.
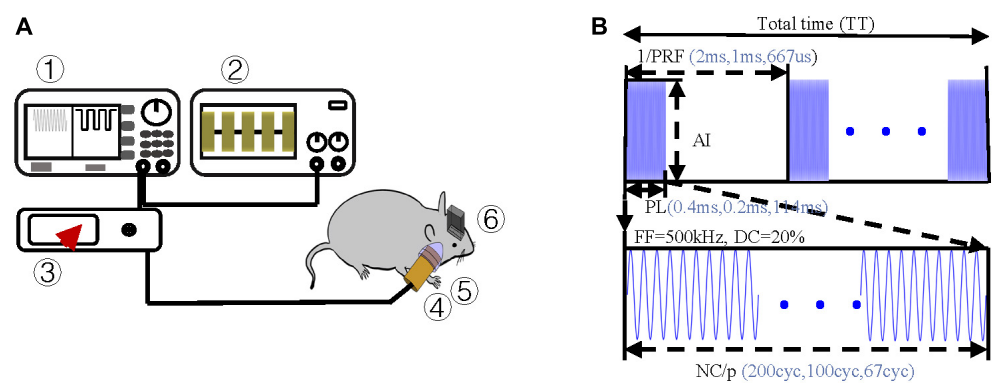

FIGURE 2 | Experimental protocol (A) Schematic of ultrasound stimulation and LFP recordings (1) arbitrary function generator, (2) oscilloscope, (3) amplifier, (4) ultrasound transducer, (5) acoustic collimator, and (6) electrodes. (B) Schematic of ultrasound sequences and associated parameters, i.e., simulation duration (SD), pulsed repetition frequency (PRF), acoustic intensity (Al), fundamental frequency (FF), and number of cycles per pulse (NC/p).

TABLE 1 | Number of nuclei showed auto-rhythm during VLTUS.

\begin{tabular}{|c|c|c|c|c|}
\hline$I_{S P P A}\left(W / c^{2}\right)$ & $I_{S P T A}\left(\mathrm{~mW} / \mathrm{cm}^{2}\right)$ & $P R F=500 \mathrm{HzNC} / \mathrm{p}=200$ & $\mathrm{PRF}=1 \mathrm{kHzNC} / \mathrm{p}=100$ & $\mathrm{PRF}=1.5 \mathrm{kHzNC} / \mathrm{p}=67$ \\
\hline 0.044 & 8.8 & 0 & 0 & 0 \\
\hline 0.082 & 16.4 & 5 & 3 & 0 \\
\hline
\end{tabular}

ISPPA: the spatial peak, pulse average intensity; ISPTA: spatial peak temporal average intensity; PRF: pulse repetition frequency; NC/p: number of cycles per pulse.

(Babadi and Brown, 2014). These powers were then normalized by mean power for the global spectrum $(1-140 \mathrm{~Hz})$ within each time serial.

To observe the time-variant spectral components of LFP signals over whole periods, TFA techniques are needed to study such non-stationary signals in both the time and frequency domains simultaneously. The S-transform expresses the phase information by using the Fourier kernel, and translates the amplitude envelope of the Gaussian window. The S-transform of a time-varying LFP signal $\mathrm{x}(\mathrm{t})$ is defined as:

$$
S_{x}(t, f)=\frac{|f|}{\sqrt{2 \pi}} \int_{-\infty}^{\infty} x(\tau) e^{\frac{-(\tau-t)^{2} f^{2}}{2}} e^{-i 2 \pi f \tau} d \tau
$$

\section{Relative Power Analysis of Different Rhythms}

The relative power represented a key power of neuromodulation parameter (Henrie and Shapley, 2005). The upper frequency cutoff of the LFP is often considered to be around $200 \mathrm{~Hz}$. In our study, after spectrum estimation of LFP signals, signals were decomposed into the delta $(1-3 \mathrm{~Hz})$, theta $(4-8 \mathrm{~Hz})$, alpha $(8-12 \mathrm{~Hz})$, beta $(12-30 \mathrm{~Hz})$, slow gamma $(30-60 \mathrm{~Hz})$, high gamma $(60-200 \mathrm{~Hz})$ frequency bands by the discrete wavelet transform (DWT) according to the rhythms (Buzsaki, 2006). Relative power was used to eliminate the difference between experiments. The relative power of each rhythm is equal to the corresponding band-limited power corrected by the global spectrum (1-200 Hz) power. For example, the band-limited power of the alpha rhythm is calculated as the spectral estimation averaged from all frequency points within the range of $8-12 \mathrm{~Hz}$, and the relative power is the ratio between the alpha power and the total power of the LFP signal.

\section{RESULTS}

\section{Neuromodulation Effect of Five Nuclei Under Different VLUTS Parameters}

Enhanced neural oscillation (the average amplitude of LFPs were 4-10 times higher than normal) with rhythmic patterns appeared 
in selected nuclei during VLUTS with PRF of 500 and $1 \mathrm{kHz}$, when the $I_{S P T A}$ raised to $12 \mathrm{~mW} / \mathrm{cm}^{2}$ (Table 1 and Figure 3 ). Under continuous ultrasonic stimulation, enhanced voltage presented such increase-subside rhythm repeats periodically with very slow frequency, around $0.03-0.05 \mathrm{~Hz}$, which bears some similarity to autorhythmicity. We define these types of oscillations pattern to be auto-rhythm. As shown in Figure 3A, the red line marks one start and end period of CA2 nucleus rhythm. The number of responding nuclei reached maximum when ISPTA raised to $16.4 \mathrm{~mW} / \mathrm{cm}^{2}$ for PRF $500 \mathrm{~Hz}$ and $20.8 \mathrm{~mW} / \mathrm{cm}^{2}$ for PRF $1 \mathrm{kHz}$ (Table 1). And the number of responding nuclei started to decrease while $\mathrm{I}_{S P T A}$ continued to increase. All such auto-rhythmic oscillations were highlighted with shadows (Figures 3A,B). The average amplitude of LFP showed in Figure 3 was listed in Table 2. Under PRF $1.5 \mathrm{kHz}$, neural oscillation was moderately increased during stimulation but without the auto-rhythm.

The LFP signal is a continuous stochastic process. It consists of a series of continuously varying voltages in time, which encodes rich information of neural activities and can be quantitatively characterized in the frequency-domain by spectral estimation. The LFP signals of individual channels before and after the ultrasound stimulation were processed by the multitaper spectral estimation technique as illustrated in Figure 3. Compared to neuronal activity prior to ultrasound stimulation, all five nuclei maintained higher excitability with the auto-rhythmic pattern during VLTUS with PRF $500 \mathrm{~Hz}, I_{S P T A}=16.4 \mathrm{~mW} / \mathrm{cm}^{2}$ and PRF $1 \mathrm{kHz}, I_{S P T A}=20.8 \mathrm{~mW} / \mathrm{cm}^{2}$, and increased highfrequency energy (slow gamma and high gamma) was observed. Intriguingly, the CA1 nuclei didn't show auto-rhythmic pattern during VLTUS with PRF $1 \mathrm{kHz}$ (Figure 3B). Under PRF $1.5 \mathrm{kHz}$ stimulation, the low frequency delta wave $(4 \mathrm{~Hz})$ was dramatically increased (Figure 3C).

Table 1 shows numbers of nuclei which showed significant neuromodulations with auto-rhythmic pattern under different UTS parameters. Under PRF $500 \mathrm{~Hz}$, all five nuclei showed the positive response at $I_{S P T A}=16.4 \mathrm{~mW} / \mathrm{cm}^{2}$. Under PRF $1 \mathrm{kHz}$, four nuclei responded at $I_{S P T A}=20.8 \mathrm{~mW} / \mathrm{cm}^{2}$. With increase the $\mathrm{AI}$, the number of responding nuclei started dropping. When $I_{S P T A}$ raised to $25.6 \mathrm{~mW} / \mathrm{cm}^{2}$ only two nuclei under PRF $500 \mathrm{~Hz}$, three nuclei under PRF $1 \mathrm{kHz}$ showed positive response. Under PRF $1.5 \mathrm{kHz}$, no auto-rhythmic pattern has been observed.

We measured the average power before and after stimulation (10 s each). Results are shown in Table 2. The average power of normal LFPs was within $0.07 \mu \mathrm{W}$, but when PRF was $500 \mathrm{~Hz}$ and $1 \mathrm{kHz}$, the average power of LFPs increased by 13-40 times. It can be seen that the transcranial ultrasound induced neurons to open ion channels and discharge. When the PRF is $1.5 \mathrm{kHz}$, the power slightly increases with obvious group slow wave, but there is no rhythmic pattern. Definitions of ultrasound parameters please see Table 3.

We utilized the TFA technology to analyze these phenomena further (Figure 4). To avoid high computational complexity, we only analyzed the LFP signal of five nuclei in 1 epoch $(2 S)$, starting from $4 \mathrm{~s}$ after stimulation to eliminate the noise at the beginning of stimulation. The normal LFP signal energy is
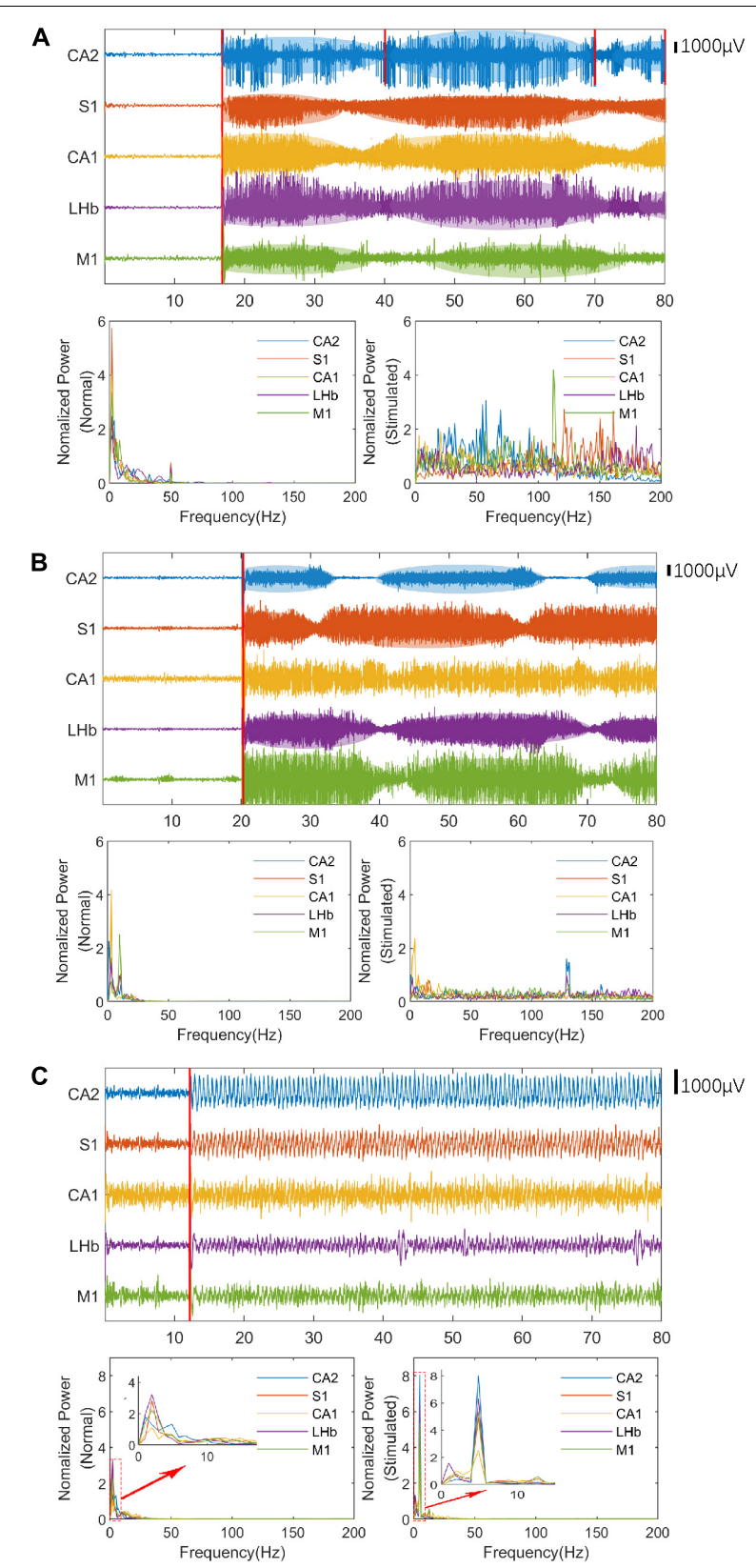

FIGURE 3 | The LFPs and spectral estimation in the rat before (normal state) and after ultrasonic stimulation. The stimulation time of ultrasound is marked with a red line. (A) The LFPs and spectral estimation with band-pass filtering $\left(P R F=500 \mathrm{~Hz}, I_{\text {SPTA }}=16.4 \mathrm{~mW} / \mathrm{cm}^{2}, 1-140 \mathrm{~Hz}\right)$ and notch filter $(50 \mathrm{~Hz})$. (B) The LFPs and spectral estimation with band-pass filtering (PRF $=1 \mathrm{kHz}$, $\left.I_{\text {SPTA }}=20.8 \mathrm{~mW} / \mathrm{cm}^{2}, 1-140 \mathrm{~Hz}\right)$ and notch filter $(50 \mathrm{~Hz})$. (C) The LFPs and spectral estimation with band-pass filtering (PRF $=1.5 \mathrm{kHz}$,

$I_{\text {SPTA }}=8.8 \mathrm{~mW} / \mathrm{cm}^{2}, 1-45 \mathrm{~Hz}$ ). Red rectangles indicate the period expanded in the small window.

mainly concentrated between $2 \mathrm{~Hz}$ and $45 \mathrm{~Hz}$. After stimulation, the high frequency component $(60-200 \mathrm{~Hz})$ of the LFP is prominent when $\mathrm{PRF}=500 \mathrm{~Hz}$ and $1 \mathrm{kHz}$, and the low frequency $(1-8 \mathrm{~Hz})$ appear and dominant when $\mathrm{PRF}=1.5 \mathrm{kHz}$. 
TABLE 2 | Average Power of LFPs before and after stimulation.

\begin{tabular}{|c|c|c|c|c|c|c|}
\hline \multirow[t]{2}{*}{ Units: $\mu$ W } & \multicolumn{2}{|c|}{ PRF $=500 \mathrm{~Hz}$} & \multicolumn{2}{|c|}{$P R F=1 \mathrm{kHz}$} & \multicolumn{2}{|c|}{ PRF = $1.5 \mathrm{kHz}$} \\
\hline & Normal & Stimulation & Normal & Stimulation & Normal & Stimulation \\
\hline CA2 & 0.018 & 0.769 & 0.008 & 0.111 & 0.014 & 0.204 \\
\hline S1 & 0.018 & 0.503 & 0.011 & 0.835 & 0.021 & 0.116 \\
\hline CA1 & 0.020 & 0.898 & 0.053 & 0.436 & 0.067 & 0.097 \\
\hline $\mathrm{LHb}$ & 0.009 & 0.749 & 0.006 & 0.475 & 0.012 & 0.047 \\
\hline M1 & 0.023 & 0.268 & 0.025 & 0.905 & 0.047 & 0.057 \\
\hline
\end{tabular}

TABLE 3 | Definitions of ultrasound parameters.

\begin{tabular}{lcc}
\hline Parameter & Abbreviation & Unit \\
\hline Fundamental frequency & $\mathrm{FF}$ & $\mathrm{kHz}$ \\
Intensity: spatial-peak, pulse-averaged & $I_{S P P A}$ & $\mathrm{~W} / \mathrm{cm}^{2}$ \\
Intensity: spatial-peak, temporal-averaged & ISPTA & $\mathrm{mW} / \mathrm{cm}^{2}$ \\
Pulse length & $\mathrm{PL}$ & $\mathrm{ms}$ \\
Pulse repetition frequency & $\mathrm{PRF}$ & $\mathrm{Hz}$ \\
Burst duty cycle & $\mathrm{BDC}$ & $\%$ \\
Duty cycle & $\mathrm{DC}$ & $\%$ \\
Number of cycles per pulse & $\mathrm{NC} / \mathrm{p}$ & - \\
Total time & $\mathrm{TT}$ & $\mathrm{s}$ \\
Mechanical index & $\mathrm{Ml}$ & - \\
Anterior-posterior & $\mathrm{AP}$ & \\
Medial-lateral & $\mathrm{ML}$ & \\
Dorsal-ventral & $\mathrm{DV}$ & \\
\hline
\end{tabular}

These phenomena are consistent with the results discussed in Figure 3.

\section{Relative Power of Different Rhythms During TUS With Different PRF}

The baseline of the stimulation or inhibition is calculated by 6 s normal LFPs (P0: reference power of normal LFPs). And LFP signals within three epochs (6 s) just $4 \mathrm{~s}$ after stimulation (to eliminate abnormal interference of brain at the beginning of stimulation) are used to analyze the relative power. To demonstrate the relative stimulation or inhibition effect of different PRF on each rhythm (compared with normal LFP signal without stimulation), the relative power of LFP signals was divided by the relative power of normal LFP signals, and the logarithm of the result was taken.

As we can see from Figure 5 when PRF $500 \mathrm{~Hz}$ or PRF $1 \mathrm{kHz}$ the gamma component of the LFP, especially high-gamma component of the LFP (hgLFP), was significantly increased after stimulation, the theta and delta power decreased otherwise. However, when PRF $1.5 \mathrm{kHz}$ high frequency band are suppressed while delta rhythms increased.

Figure 6 analyzed LFP signal of CA2 nucleus in one epoch (2S) just $4 \mathrm{~s}$ after stimulation as an example. Figure 6A represents the decomposition of the LFP signal of CA2 nucleus into different rhythms. And we analyzed the power ration of delta and high-gamma rhythms which is changed obviously in Figure 5 under different stimulation parameters. The highgamma power ratio (Figures 6B,C) was significantly increased after stimulation, while the theta power suppressed. When PRF $1.5 \mathrm{kHz}$ (Figure 6D) the powers ration of the theta and delta frequency bands were increased obvious, however, while the high-gamma power ratio remains the same.

Here we convincingly prove that the hgLFP is active when PRF $500 \mathrm{~Hz}$ and $1 \mathrm{kHz}$. And the awakening of delta and theta rhythm is a particular phenomenon when PRF $1.5 \mathrm{kHz}$.

\section{DISCUSSION}

The VLTUS exerted strong neuromodulation effect in our study, leading us to hypothesize that the membrane gating kinetics modulated by VLTUS may induce strong synchronized synaptic currents. In fact, The mechanical interaction between US and neuronal membranes can modify the membrane gating kinetics through the action on mechanosensitive voltage-gated ion channels or neurotransmitter receptors (Sukharev and Corey, 2004; Morris and Juranka, 2007; Tyler et al., 2008). We observed rhythmic high-amplitude LFP activities during low frequency and low power TUS (PRF $500 \mathrm{~Hz}$ and $1 \mathrm{kHz}$, $I_{S P T A} 12-25.6 \mathrm{~mW} / \mathrm{cm}^{2}$ ) with high frequency energy. The normal amplitude of the LFP can range from a few microvolts to hundreds of microvolts in the cortex (Goldberg et al., 2004). However, much higher LFP amplitude $(>1,000 \mu \mathrm{V})$ was observed in this study during VLTUS. LFPs represent the summed voltage fluctuations among a population of cells within a given field and are measured with electrodes. The amplitude of LFP signals is determined by correlated activities of the neurons that receive the inputs at the recording site (Goldberg et al., 2004). It indicates that synchrony of synaptic currents is enhanced during VLTUS. The underlying physiology remains unclear.

\section{The Influence of PRF Frequency on the Neural Activity}

To the best of our knowledge, this is the first report of neurons sensitive to the specific frequency and AI during ultrasound stimulation. And we could find that neurons responding selectively to inputs of a specific frequency.

When PRF $500 \mathrm{~Hz}$ or PRF $1 \mathrm{kHz}$, neurons show prefer at gamma frequencies. The increased power of hgLFP during VLTUS is a kind of neural excitement. The physiological 


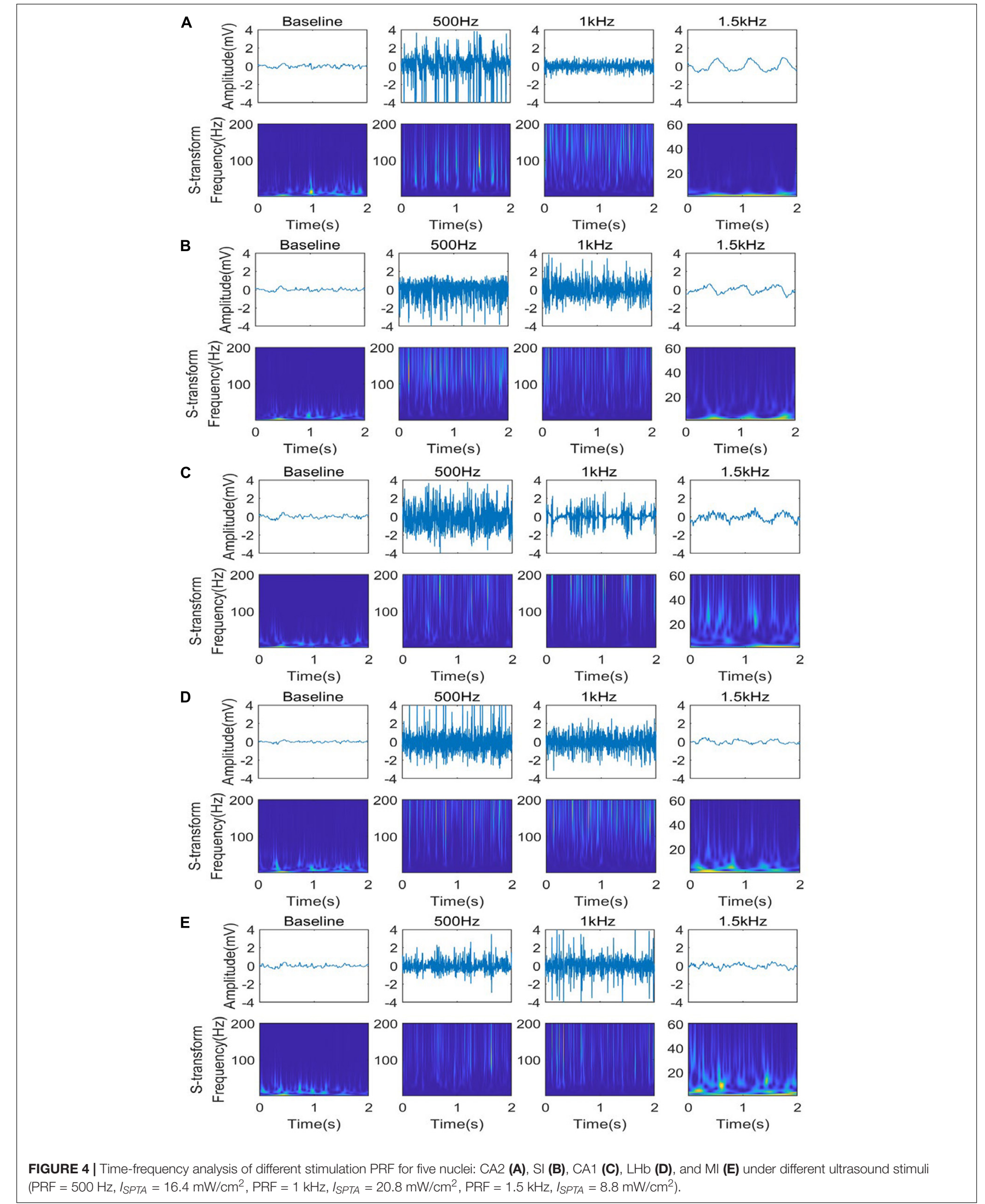



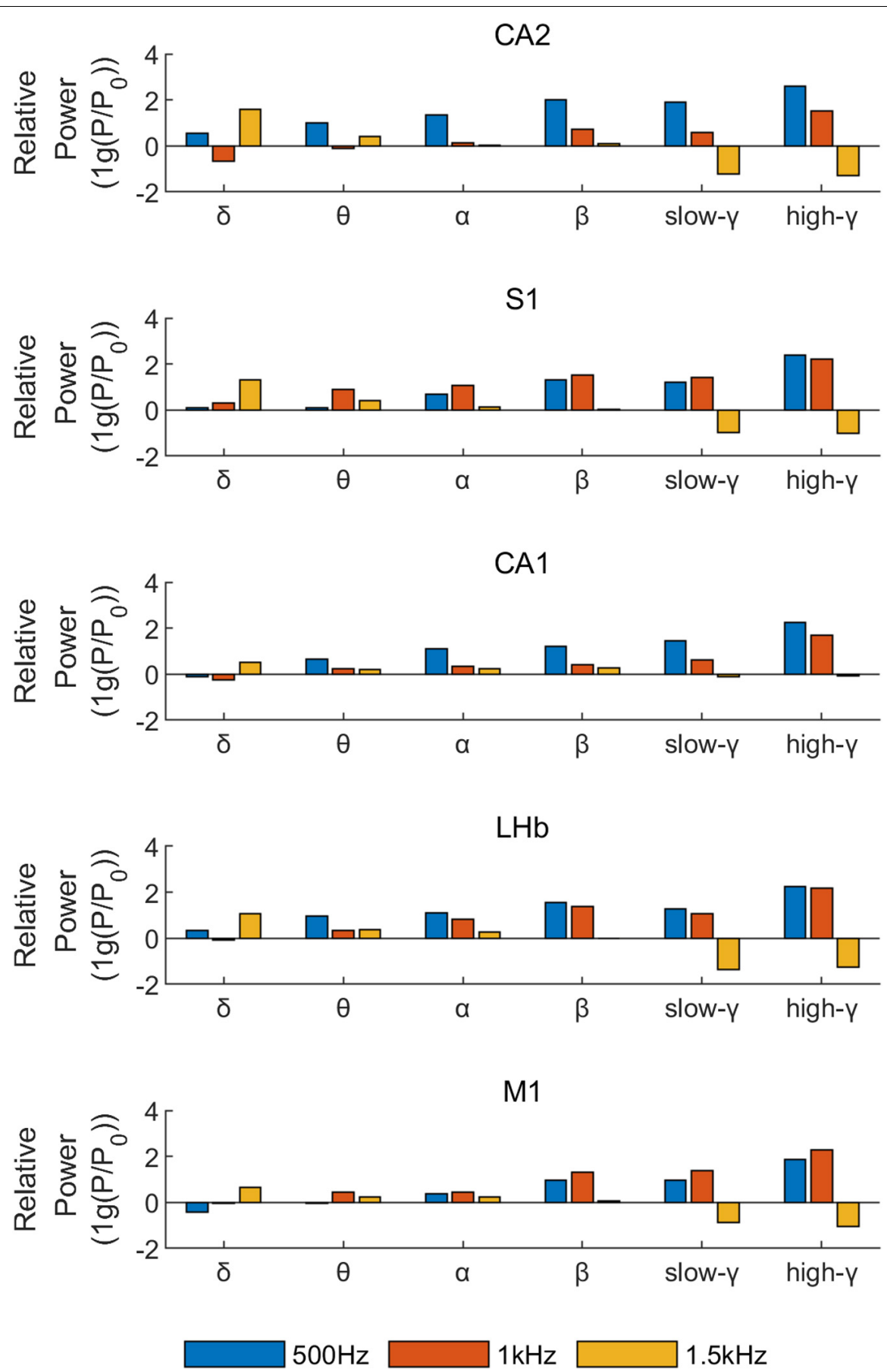

FIGURE 5 | The relative power of different rhythms for five nuclei under different ultrasound stimuli $\left(\mathrm{PRF}=500 \mathrm{~Hz}, I_{S P T A}=16.4 \mathrm{~mW} / \mathrm{cm}{ }^{2}, \mathrm{PRF}=1 \mathrm{kHz}\right.$, $\left.I_{S P T A}=20.8 \mathrm{~mW} / \mathrm{cm}^{2}, \mathrm{PRF}=1.5 \mathrm{kHz}, I_{S P T A}=8.8 \mathrm{~mW} / \mathrm{cm}^{2}\right)$.

reasons for the elevated power of hgLFP phenomenon are not clear, but previous studies can provide some insights for its source. One potential explanation is that high-frequency (>60 Hz) components of signals represent activation of neuronal populations in the underlying cortex (Crone et al., 2006). Contamination of spike waveforms from nearby cells, either too small or too variable to be detected as single unit spikes (Berens et al., 2008). TUS under PRF $500 \mathrm{~Hz}$ or $1 \mathrm{kHz}$ could wake up and activate hgLFP. Recent data recorded from animals and humans have suggested that gamma-frequency activity plays an important role in both attention and long-term memory (Jensen et al., 2007). Such hgLFP with auto-rhythm performance could 


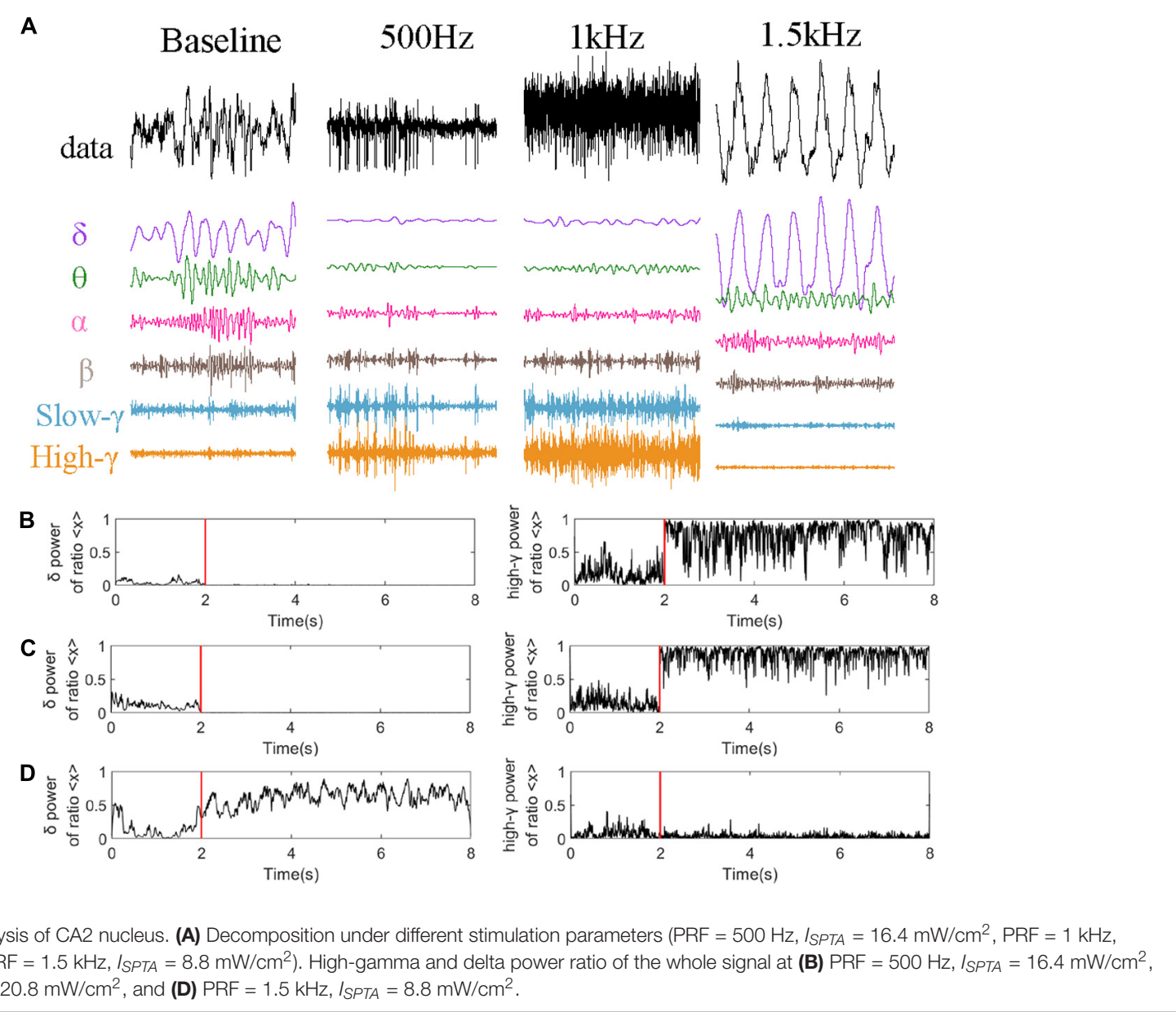

FIGURE $6 \mid$ Rhythm analysis of CA2 nucleus. (A) Decomposition under different stimulation parameters $\left(\mathrm{PRF}=500 \mathrm{~Hz}, I_{\mathrm{SPTA}}=16.4 \mathrm{~mW} / \mathrm{cm}{ }^{2}, \mathrm{PRF}=1 \mathrm{kHz}\right.$,
$\left.I_{\text {SPTA }}=20.8 \mathrm{~mW} / \mathrm{cm}^{2}, \mathrm{PRF}=1.5 \mathrm{kHz}, I_{\text {SPTA }}=8.8 \mathrm{~mW} / \mathrm{cm}^{2}\right)$. High-gamma and delta power ratio of the whole signal at $(\mathbf{B}) \mathrm{PRF}=500 \mathrm{~Hz}, I_{\text {SPTA }}=16.4 \mathrm{~mW} / \mathrm{cm}^{2}$, (C) $P R F=1 \mathrm{kHz}, I_{S P T A}=20.8 \mathrm{~mW} / \mathrm{cm}^{2}$, and (D) PRF $=1.5 \mathrm{kHz}, I_{S P T A}=8.8 \mathrm{~mW} / \mathrm{cm}^{2}$

aid in understanding neuronal processing in complex cognitive functions in future studies.

The slow waves (theta and delta frequency band) appear and occupy when PRF is $1.5 \mathrm{kHz}$. Theta rhythms are produced by local interactions between hippocampal interneurons and pyramidal cells. There is evidence that mammalian brain display intrinsic resonance with frequency selectivity for electrical stimulation inputs within the theta-range $(4-10 \mathrm{~Hz})$ (Hutcheon and Yarom, 2000; Vera et al., 2017). Seidenbecher et al. (2003) have found that synchronization of theta activities in the amygdalohippocampal network represents a neuronal correlate of conditioned fear, apt to improve neuronal communication during memory retrieval. This may be related to the fact that ultrasound causes abnormal neuronal activity, so neuronal resonance of theta and delta rhythms might be waked up, relevant to cognitive operations and learning in the brain.

\section{The Mechanism of Auto-Rhythmic Pattern}

The mechanism of auto-rhythmic pattern of neural oscillation during VLTUS remains unknown but could relate to the refractory period observed in neurons. Refractory periods are caused by the inactivation property of voltage-gated sodium channels and the lag of potassium channels in closing. Voltage-gated sodium channels have two gating mechanisms, the activation mechanism that opens the channel with depolarization, and the inactivation mechanism that closes the channel with repolarization. While the channel is in the inactive state, it will not open in response to depolarizing stimuli. LFP reflects the neural population activity. The synchronization of refractory periods amongst groups of neurons changes population neuronal activity between depolarized and hyperpolarized phases. This may cause that rhythmic pattern. Interestingly, the rhythmic patterns in five nuclei are not identical (Figure 3), which may be due to physiological features of the neuron and the characteristics of the extracellular field in each nucleus.

All five nuclei showed the auto-rhythmic neuromodulation with high frequency energy during the VLTUS (PRF $500 \mathrm{~Hz}$, $I_{S P T A} 16.4 \mathrm{~mW} / \mathrm{cm}^{2}$ ). All nuclei are in distinct brain networks and located distally, while focus ultrasound stimulation is localized. It's hard to conclude that all electrical signals were from post synaptic action. Previous studies showed that spatiotemporal fluctuations of the extracellular field may influence via purely electrostatic, so-called ephaptic coupling (Jefferys, 1995; Weiss and Faber, 2010). Network oscillations across and within brain areas are critical for brain function (Roach et al., 2018). Coherent 
oscillations could provide such a mechanism in coordinating the timing of activity and enhancing the functional link between distant areas. Ephaptic coupling may play a big role in this procedure. Our data support those findings and indicate that the ephaptic coupling could widely exist in brain networks, which strongly entrain action potentials (Anastassiou et al., 2011). Ephaptic coupling has been shown to affect population activity during hypersynchronous epileptic discharges (Jefferys, 1995; McCormick and Contreras, 2001). However, how ephaptic coupling alters the functioning of neurons under physiological conditions remains unclear. Our data suggest that VLTUS could be a valid tool in this field.

\section{Limitation}

A larger sample study will help us better evaluate the neuromodulation effect of VLTUS; Only selective PRF and AI have been tested in this pilot study. Need further experiments to prove the mapping relationship between simulation position with the five recording nuclei; The hydrophone can be used to characterize the acoustic field with the focal length and focal length of the transducer measured accurately.

\section{CONCLUSION}

In summary, VLTUS shows a significant neuromodulation effect. Both the amplitude and frequency power of LFPs were altered during specific stimulation parameters. VLTUS could be a valid tool to study neurons' physiological conditions, including ephaptic coupling.

\section{REFERENCES}

Anastassiou, C. A., Perin, R., Markram, H., and Koch, C. (2011). Ephaptic coupling of cortical neurons. Nat. Neurosci. 14, 217-223. doi: 10.1038/nn.2727

Babadi, B., and Brown, E. N. (2014). A review of multitaper spectral analysis. IEEE Trans. Biomed. Eng. 61, 1555-1564.

Bachtold, M. R., Rinaldi, P. C., Jones, J. P., Reines, F., and Price, L. R. (1998). Focused Ultrasound Modifications of Neural Circuit Activity in a Mammalian Brain. Ultrasound Med. Biol. 24, 557-565. doi: 10.1016/S0301-5629(98)00014-3

Barker, A. T. (1999). The history and basic principles of magnetic nerve stimulation. Electroencephalogr. Clin. Neurophysiol. Suppl. 51, 3-21. doi: 10. 1201/b14174-3

Berens, P., Keliris, G. A., Ecker, A. S., Logothetis, N. K., and Tolias, A. S. (2008). Comparing the feature selectivity of the gamma-band of the local field potential and the underlying spiking activity in primate visual cortex. Front. Syst. Neurosci. 2:2.

Buzsaki, G. (2006). Rhythms of the Brain. Oxford: Oxford University Press.

Bystritsky, A., Korb, A. S., Douglas, P. K., Cohen, M. S., Melega, W. P., Mulgaonkar, A. P., et al. (2011). A review of low-intensity focused ultrasound pulsation. Brain Stimulat. 4, 125-136. doi: 10.1016/j.brs.2011.03.007

Center for Devices and Radiological Health (2019). Marketing Clearance of Diagnostic Ultrasound Systems and Transducers. Available online at: https: //www.fda.gov/regulatory-information/search-fda-guidance-documents/ marketing-clearance-diagnostic-ultrasound-systems-and-transducers (accessed June, 2019).

\section{DATA AVAILABILITY STATEMENT}

The original contributions presented in the study are included in the article/supplementary material, further inquiries can be directed to the corresponding author/s.

\section{ETHICS STATEMENT}

The animal study was reviewed and approved by the Laboratory Animal Ethical and Welfare Committee of Shandong University Cheeloo College of Medicine.

\section{AUTHOR CONTRIBUTIONS}

YLiu: acquisition of data, analysis and interpretation of data, drafting, and revising the article. YZ, WZ, and YLi: design of the experiments, analysis and interpretation of data, and revising the article. GW, CC, GZ, and ET: analyzed the data and wrote the manuscript. All authors contributed to the article and approved the submitted version.

\section{FUNDING}

The support of the Youth Program of National Natural Science Foundation of China (No. 81801689), China postdoctoral foundation (No. 2017M622215), the Fundamental Research Funds of the Central Universities, Shandong Academy of Pharmaceutical Sciences, and Qiushi Academy for Advanced Studies of Zhejiang University is gratefully acknowledged. This work was also supported by a NIH grant R01AG057848.

Crone, N. E., Sinai, A., and Korzeniewska, A. (2006). High-frequency gamma oscillations and human brain mapping with electrocorticography. Prog. Brain Res. 159, 275-295. doi: 10.1016/s0079-6123(06)59019-3

Culjat, M. O., Goldenberg, D., Tewari, P., and Singh, R. S. (2010). A review of tissue substitutes for ultrasound imaging. Ultrasound Med. Biol. 36, 861-873. doi: 10.1016/j.ultrasmedbio.2010.02.012

Goldberg, J. A., Rokni, U., Boraud, T., Vaadia, E., and Bergman, H. (2004). Spike synchronization in the cortex/basal-ganglia networks of Parkinsonian primates reflects global dynamics of the local field potentials. J. Neurosci. Off. J. Soc. Neurosci. 24, 6003-6010. doi: 10.1523/JNEUROSCI.4848-03.2004

Guo, H., Hamilton, M., Offutt, S. J., Gloeckner, C. D., Li, T., Kim, Y., et al. (2018). Ultrasound Produces Extensive Brain Activation via a Cochlear Pathway. Neuron 98, 1020.e-1030.e. doi: 10.1016/j.neuron.2018. 04.036

Guo, T., Li, H., Lv, Y., Lu, H., Niu, J., Sun, J., et al. (2015). Pulsed transcranial ultrasound stimulation immediately after the ischemic brain injury is neuroprotective. IEEE Trans. Biomed. Eng. 62, 2352-2357. doi: 10.1109/tbme. 2015.2427339

Hakimova, H., Kim, S., Chu, K., Lee, S. K., Jeong, B., and Jeon, D. (2015). Ultrasound stimulation inhibits recurrent seizures and improves behavioral outcome in an experimental model of mesial temporal lobe epilepsy. Epilepsy Behav. 49, 26-32. doi: 10.1016/j.yebeh.2015.04.008

Hayner, M., and Hynynen, K. (2001). Numerical analysis of ultrasonic transmission and absorption of oblique plane waves through the human skull. J. Acoust. Soc. Am. 110, 3319-3330. doi: 10.1121/1.1410964 
Henrie, J. A., and Shapley, R. (2005). LFP power spectra in V1 cortex: the graded effect of stimulus contrast. J. Neurophysiol. 94, 479-490. doi: 10.1152/jn.00919. 2004

Hu, L., and Zhang, Z. (2019). EEG Signal Processing and Feature Extraction. New York, NY: Springer.

Hutcheon, B., and Yarom, Y. (2000). Resonance, oscillation and the intrinsic frequency preferences of neurons. Trends Neurosci. 23, 216-222. doi: 10.1016/ S0166-2236(00)01547-2

Jefferys, J. G. (1995). Nonsynaptic modulation of neuronal activity in the brain: electric currents and extracellular ions. Physiol. Rev. 75, 689-723. doi: 10.1152/ physrev.1995.75.4.689

Jensen, O., Kaiser, J., and Lachaux, J.-P. (2007). Human gamma-frequency oscillations associated with attention and memory. Trends Neurosci. 30, 317324. doi: 10.1016/j.tins.2007.05.001

Lin, W.-T., Chen, R.-C., Lu, W.-W., Liu, S.-H., and Yang, F.-Y. (2015). Protective effects of low-intensity pulsed ultrasound on aluminuminduced cerebral damage in Alzheimer's disease rat model. Sci. Rep. 5:9671.

Matsumoto, M., and Hikosaka, O. (2009). Representation of negative motivational value in the primate lateral habenula. Nat. Neurosci. 12, 77-84. doi: 10.1038/nn. 2233

McCormick, D. A., and Contreras, D. (2001). On The Cellular and Network Bases of Epileptic Seizures. Annu. Rev. Physiol. 63, 815-846. doi: 10.1146/annurev. physiol.63.1.815

Morris, C. E., and Juranka, P. F. (2007). "“Chapter 11 - Lipid Stress at Play: Mechanosensitivity of Voltage-Gated Channels,", in Current Topics in Membranes Mechanosensitive Ion Channels, Part B, ed. O. P. Hamill (Cambridge, MA: Academic Press), 297-338. doi: 10.1016/s1063-5823(06) 59011-8

O’Brien, W. D. Jr. (2007). Ultrasound-biophysics mechanisms. Prog. Biophys. Mol. Biol. 93, 212-255. doi: 10.1016/j.pbiomolbio.2006.07.010

Paxinos, G., and Watson, C. (2006). The Rat Brain in Stereotaxic Coordinates: Hard Cover Edition. Amsterdam: Elsevier.

Perera, T., George, M. S., Grammer, G., Janicak, P. G., Pascual-Leone, A., and Wirecki, T. S. (2016). The clinical TMS society consensus review and treatment recommendations for TMS therapy for major depressive disorder. Brain Stimulat. 9, 336-346. doi: 10.1016/j.brs.2016. 03.010

Rinaldi, P. C., Jones, J. P., Reines, F., and Price, L. R. (1991). Modification by focused ultrasound pulses of electrically evoked responses from an in vitro hippocampal preparation. Brain Res. 558, 36-42. doi: 10.1016/0006-8993(91) 90711-4

Roach, J. P., Pidde, A., Katz, E., Wu, J., Ognjanovski, N., Aton, S. J., et al. (2018). Resonance with subthreshold oscillatory drive organizes activity and optimizes learning in neural networks. Proc. Natl. Acad. Sci. 115, E3017-E3025. doi: $10.1073 /$ pnas. 1716933115

Sato, T., Shapiro, M. G., and Tsao, D. Y. (2018). Ultrasonic Neuromodulation Causes Widespread Cortical Activation via an Indirect Auditory Mechanism. Neuron 98, 1031.e-1041.e. doi: 10.1016/j.neuron.2018. 05.009

Seidenbecher, T., Laxmi, T. R., Stork, O., and Pape, H.-C. (2003). Amygdalar and hippocampal theta rhythm synchronization during fear memory retrieval. Science 301, 846-850. doi: 10.1126/science.108 5818

Sukharev, S., and Corey, D. P. (2004). Mechanosensitive Channels: Multiplicity of Families and Gating Paradigms. Sci. STKE 2004, re4-re4. doi: 10.1126/stke. 2192004re4

Tufail, Y., Matyushov, A., Baldwin, N., Tauchmann, M. L., Georges, J., Yoshihiro, A., et al. (2010). Transcranial Pulsed Ultrasound Stimulates Intact Brain Circuits. Neuron 66, 681-694. doi: 10.1016/j.neuron.2010.05.008

Tyler, W. J., Tufail, Y., Finsterwald, M., Tauchmann, M. L., Olson, E. J., and Majestic, C. (2008). Remote Excitation of Neuronal Circuits Using LowIntensity, Low-Frequency Ultrasound. PLoS One 3:e3511. doi: 10.1371/journal. pone.0003511

Vera, J., Pereira, U., Reynaert, B., Bacigalupo, J., and Sanhueza, M. (2017). Neuronal resonance in the theta $(4-10 \mathrm{~Hz})$ frequency range is modulated by dynamic changes in the input resistance. bioRxiv 2017:224253. doi: 10.1101/ 224253

Wang, X., Yan, J., Wang, Z., Li, X., and Yuan, Y. (2019). Neuromodulation effects of ultrasound stimulation under different parameters on mouse motor cortex. IEEE Trans. Biomed. Eng. 67, 291-297. doi: 10.1109/tbme.2019.2912840

Wang, Z., Yan, J., Wang, X., Yuan, Y., and Li, X. (2020). Transcranial ultrasound stimulation directly influences the cortical excitability of the motor cortex in Parkinsonian mice. Mov. Disord. 35, 693-698. doi: 10.1002/mds.27952

Weiss, S. A., and Faber, D. S. (2010). Field Effects in the CNS Play Functional Roles. Front. Neural Circuits 4:00015. doi: 10.3389/fncir.2010.00015

Yoo, S.-S., Bystritsky, A., Lee, J.-H., Zhang, Y., Fischer, K., Min, B.-K., et al. (2011). Focused ultrasound modulates region-specific brain activity. Neuroimage 56, 1267-1275. doi: 10.1016/j.neuroimage.2011.02.058

Younan, Y., Deffieux, T., Larrat, B., Fink, M., Tanter, M., and Aubry, J. (2013). Influence of the pressure field distribution in transcranial ultrasonic neurostimulation. Med. Phys. 40:082902. doi: 10.1118/1.4812423

Yuan, Y., Wang, Z., Liu, M., and Shoham, S. (2020). Cortical hemodynamic responses induced by low-intensity transcranial ultrasound stimulation of mouse cortex. NeuroImage 211:116597. doi: 10.1016/j.neuroimage.2020.116597

Yuan, Y., Zhang, K., Zhang, Y., Yan, J., Wang, Z., Wang, X., et al. (2021). The effect of low-intensity transcranial ultrasound stimulation on neural oscillation and hemodynamics in the mouse visual cortex depends on anesthesia level and ultrasound intensity. IEEE Trans. Biomed. Eng. 2021:3050797.

Zhang, D., Li, H., Sun, J., Hu, W., Jin, W., Li, S., et al. (2018). Antidepressant-like effect of low-intensity transcranial ultrasound stimulation. IEEE Trans. Biomed. Eng. 66, 411-420. doi: 10.1109/tbme.2018.2845689

Conflict of Interest: The authors declare that the research was conducted in the absence of any commercial or financial relationships that could be construed as a potential conflict of interest.

Copyright (c) 2021 Liu, Wang, Cao, Zhang, Tanzi, Zhang, Zhou and Li. This is an open-access article distributed under the terms of the Creative Commons Attribution License (CC BY). The use, distribution or reproduction in other forums is permitted, provided the original author(s) and the copyright owner(s) are credited and that the original publication in this journal is cited, in accordance with accepted academic practice. No use, distribution or reproduction is permitted which does not comply with these terms. 


\section{APPENDIX}

\section{Acoustic Intensity Calculation}

Using the radiation force method, we could calculate the acoustic power. The pressure of radiation $\left(P_{\tau}\right)$ is equal to the energy density (E):

$$
P_{\tau}=E
$$

We use a precision balance and a radiation force target that is perfectly absorptive. The conversion from force to ultrasonic power can be accomplished by using a simplified equation:

$$
\mathrm{P}=\mathrm{w} \times 14.56
$$

where $\mathrm{w}$ is the ultrasonic force in grams and $p$ is the ultrasonic power in watts.

Power intensity is rated as Spatial Peak Temporal Average $\left(\mathrm{I}_{S P T A}\right)$ and Spatial Peak Pulse Average $\left(\mathrm{I}_{S P P A}\right)$. $\mathrm{I}_{S P P A}$ is defined as

$$
I_{S P P A}=\frac{\mathrm{P}}{\mathrm{S}}
$$

where $S$ is the cross-sectional area of the given transducer. And $\mathrm{I}_{S P T A}$ is defined as:

$$
I_{S P T A}=I_{S P P A} \cdot(\mathrm{DC})
$$

where DC is the duty cycle.

Brain and tissues were assumed to have the sound speed and density of water. Moreover, in order to get the acoustic intensity transmitted into the brain, the absorption and refraction characteristics in the skull was considered. The acoustic intensity (I) after passing through skull can be estimated as (O'Brien, 2007)

$$
\mathrm{I}=\mathrm{I}_{0} \mathrm{e}^{-\alpha \mathrm{l}}
$$

Where $\mathrm{I}_{0}$ is the initial intensity, $\alpha$ is the attenuation coefficient [3.5 dB/cm in rat's skull at $0.5 \mathrm{MHz}$ (Culjat et al., 2010)], and 1 is the thickness of skull of rat [about $0.5 \mathrm{~mm}$ (Younan et al., 2013)]. 